\title{
Synthesis, Crystal and Band Structures, and Optical Properties of a New Quaternary Metal Pnictidehalide: $\left(\mathrm{Hg}_{2} \mathrm{Cd}_{2} \mathrm{As}_{2} \mathrm{Br}\right) \mathrm{Br}$
}

\author{
Jou-Ping Zou, a,b Guo-Cong Guo, , ${ }^{\text {a }}$ Wen-Tong Chen, ${ }^{a}$ Xi Liu, ${ }^{a}$ Ming-Lai Fu, ${ }^{\text {a }}$ Zhang-Jing
} Zhang, ${ }^{\mathrm{a}}$ Jin-Shun Huang ${ }^{\mathrm{a}}$

${ }^{a}$ State Key Laboratory of Structural Chemistry, Fujian Institute of Research on the Structure of Matter, Chinese Academy of Sciences, Fuzhou, Fujian 350002, P. R. China

${ }^{\mathrm{b}}$ Graduate School, Chinese Academy of Sciences, Beijing 100039, P. R. China

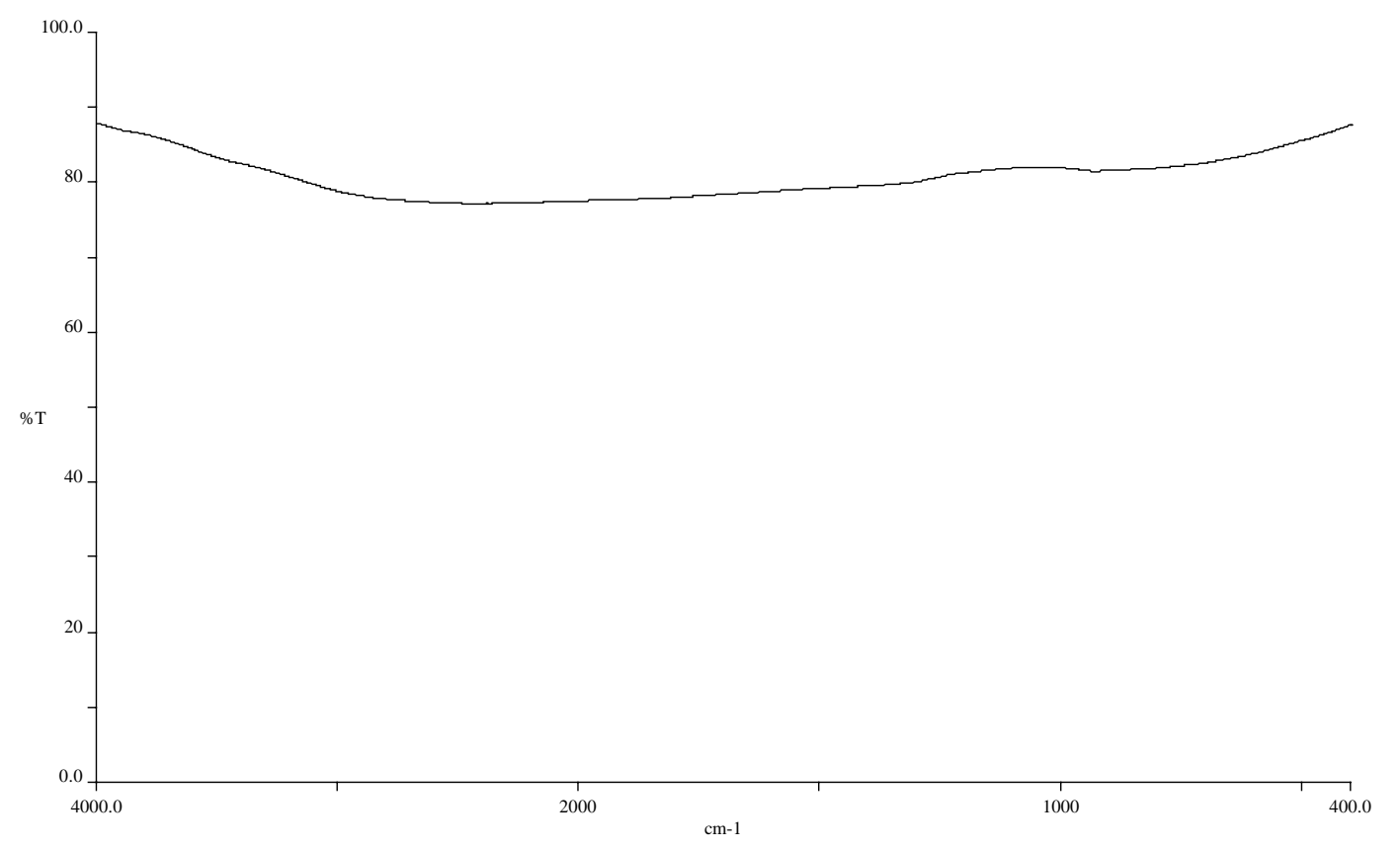

Fig. S1. FTIR spectrum of 1 on transparent pellet (about $1 \%$ sample mixed with $\mathrm{KBr}$ ).

\footnotetext{
* Corresponding author. Tel.: +86 591 83705882; fax: +86 591 83714946. E-mail address: gcguo@ms.fjirsm.ac.cn
} 


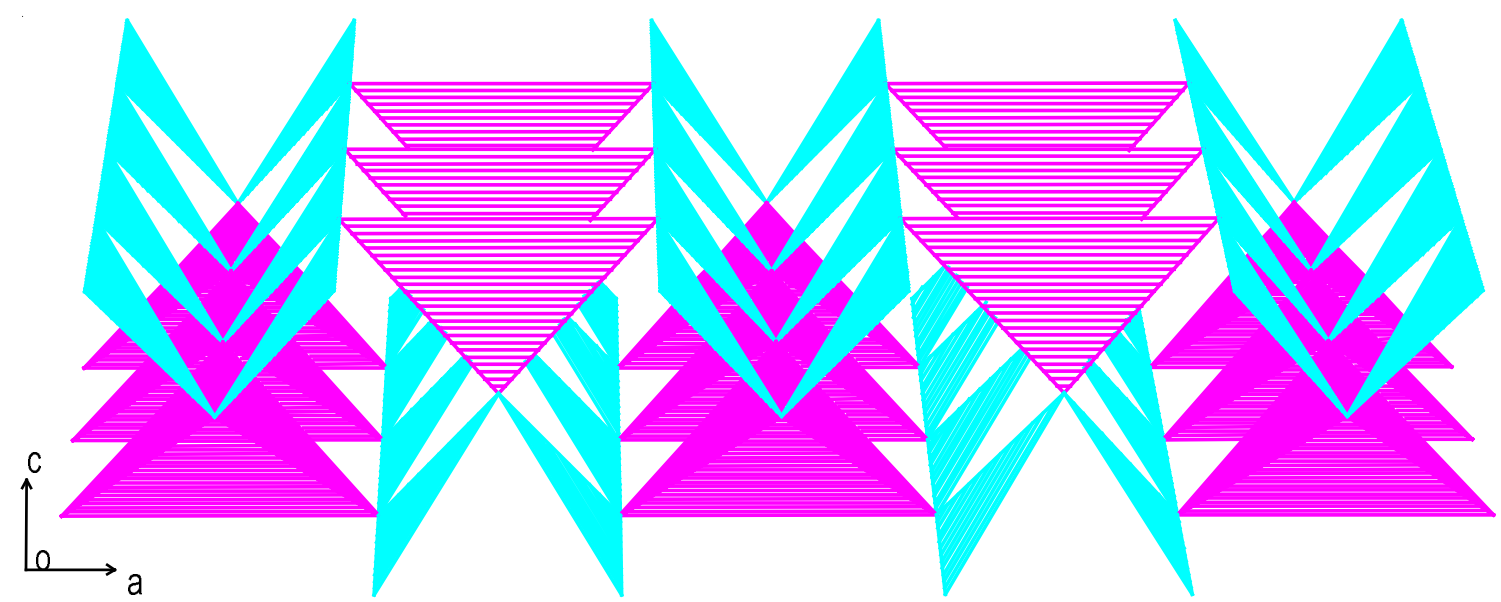

Fig. S2. A view of linear $\mathrm{Hg}$ tetrahedral chains and V-shape $\mathrm{Cd}$ trigonal chains extending along the $b$ direction.

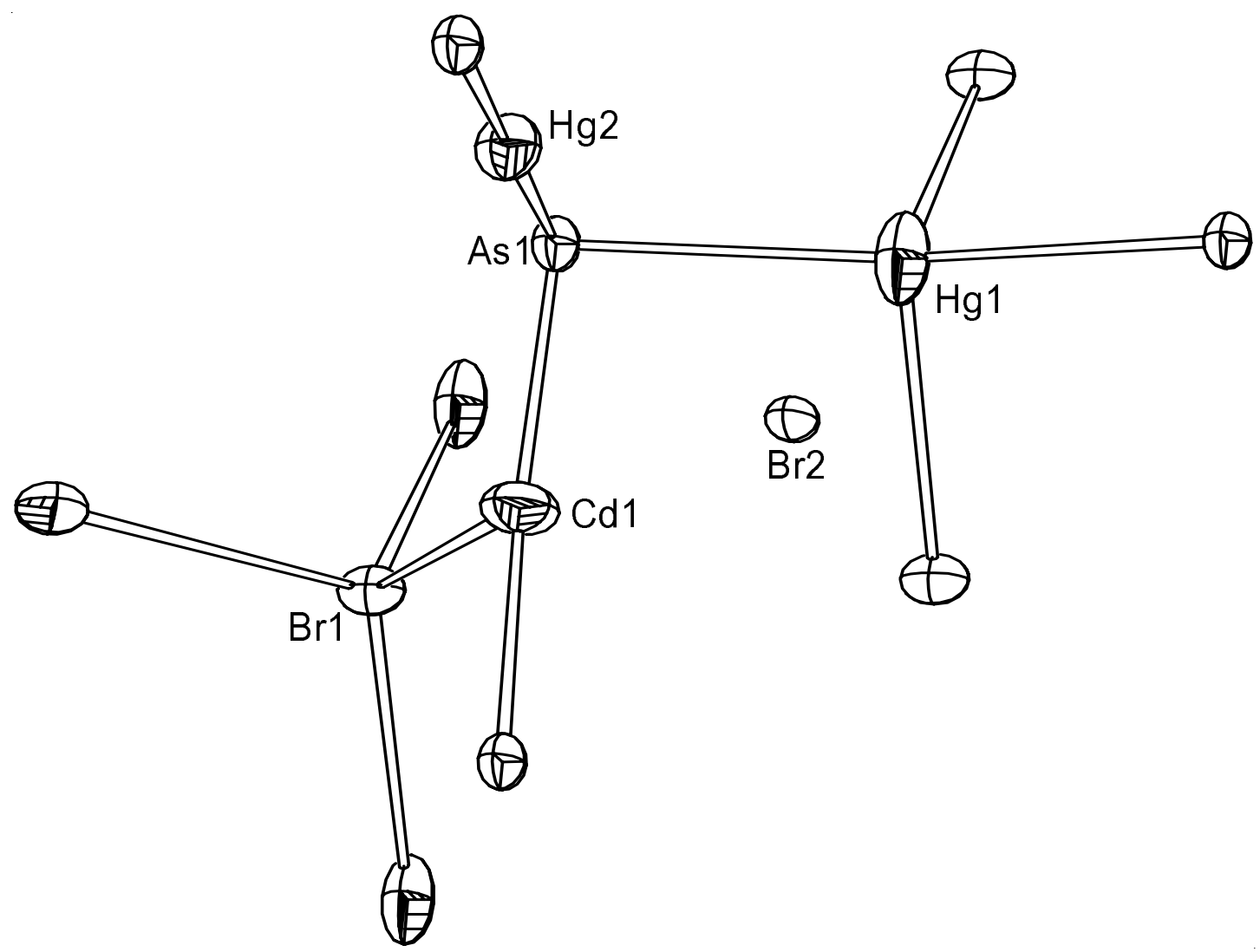

Fig. S3. Molecular structure of 1 with 30\% thermal ellipsoids. 